\title{
Video Article \\ Assessment of Ovarian Cancer Spheroid Attachment and Invasion of Mesothelial Cells in Real Time
}

\author{
Maree Bilandzic ${ }^{1}$, Kaye L. Stenvers ${ }^{1,2}$ \\ ${ }^{1}$ Reproductive Development and Cancer Laboratory, MIMR-PHI Institute of Medical Research \\ ${ }^{2}$ Department of Developmental Biology and Anatomy, Monash University
}

Correspondence to: Maree Bilandzic at maree.bilandzic@princehenrys.org

URL: https://www.jove.com/video/51655

DOI: doi: $10.3791 / 51655$

Keywords: Medicine, Issue 87, Ovarian cancer, metastasis, invasion, mesothelial cells, spheroids, real time analysis

Date Published: 5/20/2014

Citation: Bilandzic, M., Stenvers, K.L. Assessment of Ovarian Cancer Spheroid Attachment and Invasion of Mesothelial Cells in Real Time. J. Vis. Exp. (87), e51655, doi:10.3791/51655 (2014).

\section{Abstract}

Ovarian cancers metastasize by shedding into the peritoneal fluid and dispersing to distal sites within the peritoneum. Monolayer cultures do not accurately model the behaviors of cancer cells within a nonadherent environment, as cancer cells inherently aggregate into multicellular structures which contribute to the metastatic process by attaching to and invading the peritoneal lining to form secondary tumors. To model this important stage of ovarian cancer metastasis, multicellular aggregates, or spheroids, can be generated from established ovarian cancer cell lines maintained under nonadherent conditions. To mimic the peritoneal microenvironment encountered by tumor cells in vivo, a spheroidmesothelial co-culture model was established in which preformed spheroids are plated on top of a human mesothelial cell monolayer, formed over an extracellular matrix barrier. Methods were then developed using a real-time cell analyzer to conduct quantitative real time measurements of the invasive capacity of different ovarian cancer cell lines grown as spheroids. This approach allows for the continuous measurement of invasion over long periods of time, which has several advantages over traditional endpoint assays and more laborious real time microscopy image analyses. In short, this method enables a rapid, determination of factors which regulate the interactions between ovarian cancer spheroid cells invading through mesothelial and matrix barriers over time.

Video Link

The video component of this article can be found at https://www.jove.com/video/51655/

\section{Introduction}

Ovarian cancer has the highest mortality rate of all gynaecological cancers ${ }^{1}$. Worldwide, there are $\sim 230,000$ cases and over 100,000 deaths due to the disease each year ${ }^{1}$. Most patients $(>75 \%)$ are diagnosed after the cancer has already metastasized, when treatment is further complicated by increased resistance to chemotherapy and the prognosis is poor ${ }^{2}$. Patients with stage III-IV metastatic disease have 5 year survival rates of only $30-40 \%{ }^{3}$. Thus, there is an urgent need for a better understanding of the cellular behaviors underlying ovarian cancer metastasis in order to effectively treat advanced disease.

The current model of ovarian cancer metastasis proposes several steps in the metastatic process, each of which occurs in a distinct microenvironment which impacts on tumor behavior. Metastasizing ovarian cancer cells initially are shed from the primary tumor site and survive in a nonadherent state within the peritoneal fluid as single cells or three-dimensional multicellular structures, termed multicellular aggregates or spheroids $^{2,4,5}$. Cells which do not form spheroids in suspension are more susceptible to anoikis ${ }^{2,4,5}$. Spheroids also exhibit increased resistance to chemotherapeutics, contributing to residual disease and subsequent recurrence of the cancer ${ }^{2,4,5}$. A second critical step in ovarian cancer metastasis is the transition of aggregates from free-floating clusters to established secondary tumors within the peritoneal wall and omentum ${ }^{5,6}$. Cell-cell and cell-substrate interactions have been implicated in aggregate formation, survival, and adherence to the extracellular matrix (ECM) produced by the mesothelial lining of the peritoneum ${ }^{4-11}$. As yet, these processes are poorly understood.

To define the mechanisms involved in the dissemination of ovarian cancers, spheroids can be generated from established cancer cell lines using nonadherent culture methods, maintaining cells in suspension either by adding methylcellulose to the culture media ${ }^{12,13}$ or by culturing cells on low-attachment plates ${ }^{2,14}$. When cultured in this manner, ovarian cancer cells assemble into multicellular aggregates or spheroids which exhibit similar cellular, molecular, and biochemical properties to those of tumor aggregates found in vivo ${ }^{2,14}$. After formation, spheroids can be subsequently harvested from the nonadherent medium and replated onto a variety of solid surfaces for further functional studies. This represents an advance over assays in monolayer culture, which do not accurately model the behaviors of ovarian cancer cells metastasizing within a nonadherent environment such as the intraperitoneal fluid.

The invasive capacity of cancer spheroids can be assessed in traditional endpoint assays in Boyden chambers ${ }^{2}$, but this approach can be misleading, as ovarian cancer cell invasion is a dynamic process over time. A recent real-time method has been devised using time-lapse video microscopy of mesothelial cell clearance by invading ovarian cancer spheroids ${ }^{15,16}$; however, analysis of time lapse data is time consuming and can be subjective. Herein, a rapid, quantitative method for assessing the invasive behaviors of ovarian cancer spheroids in real-time is 
described. Firstly, a spheroid-mesothelial cell model was established which represents the stage of ovarian cancer metastasis when multicellular spheroids attach to and invade the peritoneal lining to form secondary tumors. Then methodology for a Real Time Cell Analyzer (RTCA; see the Materials table for company details) was adapted to conduct quantitative real time measurements of the invasive capacity of different ovarian cancer cell lines grown as spheroids and tested in this model.

In the RTCA instrument, cellular responses are monitored continuously over the course of an assay, without the need for exogenous labels, by measuring changes in electrical impedance. Specially designed culture plates have gold coated microelectrodes located underneath a microporous membrane at the interface between the upper and lower chambers of a 2 chambered well ('CIM' plates). The location of the electrodes in the lower chamber enables measurement of changes in electrical impedance as cells invade through an ECM or ECM/cellular barrier. The membrane interface between the 2 chambers of each CIM plate well is firstly coated with the ECM component of interest. In the spheroid-mesothelial cell model system, the interface is coated with a layer of Matrigel (see the Materials table for company details), to mimic the complex ECM underlying the mesothelial lining of the peritoneum, followed by a confluent monolayer of human mesothelial 'target' cells. Finally, pre-formed ovarian cancer spheroids are added. Ovarian cancer spheroid cells must actively invade through the target cells layer and matrix to reach the bottom chamber and alter electrical impedance readings. Target cells on their own are also assessed to ensure that they do not invade through the matrix layer and are suitable for use in this assay.

\section{Protocol}

\section{Preparation of Cells, Media, and Reagents}

1. Preparation of Methylcellulose-containing Medium

1. Dissolve $1.5 \mathrm{~g}$ of methylcellulose in $100 \mathrm{ml}$ of sterile Dulbecco's Modified Eagle Medium (DMEM) (no additives) in a $250 \mathrm{ml}$ flask.

2. Heat the solution on low in a microwave for $5 \mathrm{~min}$; take care to avoid boiling over.

3. Once the methylcellulose powder is semi-dissolved, add a clean magnetic stirrer and media to take the volume to $125 \mathrm{ml}$

4. Mix, invert, and stir at room temperature for $1.5 \mathrm{hr}$, followed by stirring at $4{ }^{\circ} \mathrm{C}$ overnight.

5. Divide the solution equally into four $50 \mathrm{ml}$ tubes and centrifuge for $1.5 \mathrm{hr}$ at $2,300 \mathrm{xg}$.

6. Collect the clear, highly viscous supernatant (approximately $90-95 \%$ of stock), aliquot into sterile $50 \mathrm{ml}$ tubes, and store at $4{ }^{\circ} \mathrm{C}$ for up to 3 months

2. Culture and Labeling of Cells

1. Maintain ovarian cancer cell lines in monolayer in a tissue culture incubator at $37{ }^{\circ} \mathrm{C}, 5 \% \mathrm{CO}_{2}$ in appropriate growth media (DMEM for the KGN cells ${ }^{17}$ and MCBD110:M199 for the OVCA429 and OVCA433 cell lines ${ }^{18}$ ) supplemented with $10 \%$ fetal bovine serum (FBS), 2 $\mathrm{mM}$ L-glutamine, and $1 \%$ penicillin-streptomycin.

2. Maintain LP9 human mesothelial cells in M199:Hams F12 media containing 15\% FBS, $10 \mathrm{ng} / \mathrm{ml}$ Epidermal Growth Factor (EGF) and $400 \mathrm{ng} / \mathrm{ml}$ hydrocortisone, in an incubator at $37^{\circ} \mathrm{C}, 5 \% \mathrm{CO}_{2}$.

3. Seed cells in the desired flask size and grow to approximately $75 \%$ confluency. Harvest cells by trypsinization using $0.05 \%$ trypsin/ EDTA, spin at $186 \mathrm{xg}$ for $5 \mathrm{~min}$, and wash cell pellet twice with PBS.

4. Count cells using a hemocytometer.

3. Optional Fluorescent Cell Labeling Method

1. Resuspend cancer cells at a final concentration of $1 \times 10^{6} \mathrm{cells} / \mathrm{ml}$ in $1 \mathrm{ml}$ of prewarmed PBS $/ 0.1 \%$ BSA.

2. Add $2 \mu \mathrm{l}$ of $5 \mathrm{mM}$ stock Cell Trace CSFE solution (or other preferred cell label which is retained long-term) to cells at a final concentration of $10 \mu \mathrm{M}$ and incubate at $37^{\circ} \mathrm{C}$ for $10-15 \mathrm{~min}$ in a waterbath.

3. Quench the reaction with $1 \mathrm{ml}$ of ice cold medium containing $10 \%$ FBS and re-pellet by centrifugation. Resuspend in $10 \mathrm{ml}$ of the appropriate prewarmed growth medium (see Step 1.2.1).

4. Seed cells into a $75 \mathrm{~cm}^{2}$ filter-capped flask and culture at $37{ }^{\circ} \mathrm{C}, 5 \% \mathrm{CO}_{2}$.

5. Check cells under a fluorescence microscope to verify that the level of fluorescence labeling is adequate for detection. Once cells reach approximately $75 \%$ confluency, harvest and count as in steps 1.2 .3 and 1.2.4 above.

\section{Generation of Spheroids in Methylcellulose}

1. Calculate the volume of ovarian cancer cells from Section 1 needed for spheroid generation (a total of 300,000 cells are required for each full 96-well plate experiment). Note: if using different cell lines than presented here, cell densities for uniform spheroid generation may need to be optimized for each line.

2. To calculate dilution of cells in methylcellulose media, firstly subtract the cell volume required (step 2.1 ) from $15 \mathrm{ml}$.

3. Add $3 \mathrm{ml}$ of methylcellulose stock solution (to make $20 \%$ methylcellulose final) to a volume of culture medium appropriate for each cell line, equal to $15 \mathrm{ml}$ minus the cell volume and mix thoroughly by gentle inversion.

4. Add 300,000 cells to the methylcellulose containing medium and mixed thoroughly by gentle inversion.

5. Pipette $150 \mu \mathrm{l}$ of the cell/methylcellulose/media mix (for a total of 3,000 cells/well) into each well of a 96 -well concave bottom culture plate and culture at $37^{\circ} \mathrm{C}, 5 \% \mathrm{CO}_{2}$ for $1-4$ days or until uniform spheroids form ( 1 spheroid/well is typically observed).

\section{RTCA Cell Invasion Assays}

NOTE: Impedance readings are expressed as Cell Index $(\mathrm{Cl})$, a dimensionless parameter which is a relative change in measured cell impedance representing the cell status. For analysis, import data into a spreadsheet, such as Microsoft Excel.

1. Plate Preparation 
1. Working in groups of 4 wells at a time, add $50 \mu \mathrm{l}$ matrix (diluted 1:10 in serum-free culture media) to each well of the upper chamber of a 16-well RTCA CIM plate to ensure that the total surface area is covered. Remove $30 \mu \mathrm{l}$ of matrix immediately, but slowly, to get rid of any excess fluid.

2. Incubate plate at $37^{\circ} \mathrm{C}$ for $4 \mathrm{hr}$ prior to use in a tissue culture incubator.

3. Add $30 \mu \mathrm{l}$ of the serum-free medium (SFM) appropriate for each cancer cell line to the upper chamber and $160 \mu \mathrm{l}$ of media with or without serum (as per your experimental design) to the lower chamber.

4. Assemble the $\mathrm{CIM}$ plate by clicking the lower chamber into the upper chamber and equilibrate in a $37^{\circ} \mathrm{C}$ incubator for $1 \mathrm{hr}$ in a tissue culture incubator.

2. Programming the RTCA Instrument

1. Open the RTCA program and select the 'Layout' tab. Highlight all experimental wells.

2. Right click on wells and select 'Turn on wells'; then fill in experimental conditions and cell names as desired.

3. To add steps or substeps to the program, select the 'Schedule' tab and right click on 'Add a step'. The first step is a preprogrammed background sweep, which is automatically incorporated into the program once 'add a step' is chosen.

4. Enter the desired program details for Step 2 of the RTCA program, which will record readings during the establishment of the LP9 monolayer. Add the time intervals between impedance readings by selecting the 'Interval' tab and entering ' 15 min'. Add experimental duration by selecting the 'Duration' tab and entering a time between 12-24 $\mathrm{hr}$.

5. To add subsequent steps, select the 'Schedule' tab and right click on 'Add a step' and enter details of the additional steps, as above. Step 3 of the RTCA program will direct the instrument to take readings during spheroid invasion; enter ' 5 min' under the 'Interval' tab and '48 $\mathrm{hr}$ ' under the 'Duration' tab.

6. Select 'Plate' on the menu bar and save.

3. Generation of LP9 Monolayer and Measurement of Spheroid Invasion

1. Place CIM plate in RTCA instrument and open program from Step 3.2 above. Prior to adding cells, select 'Execute' in the menu bar and then 'Start'. A background sweep will automatically be performed (Step 1 in the RTCA software instructions).

2. Remove the CIM plate from the instrument following the background sweep and place in a tissue culture hood.

3. Plate $50,000 \mathrm{LP9}$ cells suspended in $160 \mu \mathrm{l} \mathrm{SFM}$ into the upper chamber of each CIM plate well.

4. Place the plate in the RTCA instrument and take readings every 15 min while the LP9 monolayer is establishing overnight (Step 2 of the RTCA program).

5. Harvest cancer spheroids generated under Step 2 'Generation of spheroids in methylcellulose', above.

1. Aseptically, cut $1 \mathrm{~mm}$ off the top of a $1 \mathrm{ml}$ pipette tip and use to gently retrieve the contents of each well. Transfer to a sterile tube

2. Centrifuge spheroids at $120 \times \mathrm{g}$ for $8 \mathrm{~min}$, and remove methylcellulose containing medium by gentle aspiration.

3. Wash spheroids twice more with PBS, centrifuging at $120 \mathrm{xg}$ for $8 \mathrm{~min}$ to pellet spheroids after each wash.

4. For each experimental well, resuspend a total of 10 spheroids in $160 \mu \mathrm{l}$ of medium without FBS.

5. Pause the RTCA experiment by selecting 'Execute' from the menu bar and checking 'Pause'. Remove the CIM plate from the instrument, and place in a tissue culture hood. Aspirate off the media from each well and replace with spheroid-containing media (10 spheres in $160 \mu \mathrm{l}$ ) or fresh media for LP9-only control wells.

6. Return the plate to the RTCA instrument. Select 'Execute' from the menu bar and check 'Abort step'. The program will move to the next step automatically. To resume the experiment, select 'Execute' from the menu bar and check 'Start/Continue'. Step 3 in the RTCA program will initiate.

4. Data Analysis

1. To determine the level of spheroid cell invasiveness, subtract the values obtained for the LP9 monolayer only from the individual readings obtained for the ovarian cancer cell lines at each timepoint.

2. To plot 'spheroid invasion', normalize all values to the time point at which the spheroids were added to the plate, by setting that time point to ' 0 '.

\section{Representative Results}

Ovarian cancer spheroids can be generated from many types of cell lines by culturing them in suspension or by harvesting primary tumor cells from malignant ascites ${ }^{2,14}$. Here two epithelial ovarian cancer cell lines, OVCA433 and OVCA429, and an ovarian granulosa cell tumor line, KGN, have been used to generate spheroids (Figure 1A). In this method, cells are cultured in U-bottomed wells while suspended in media which has been made viscous by the addition of methylcellulose. Under these conditions, many ovarian cancer cells exhibit an inherent ability to aggregate to form spheroids ${ }^{13}$. Following overnight culture suspended in methylcellulose/media, all three cell lines formed compact spheroid structures of approximately 400-500 $\mu \mathrm{m}$ in diameter (Figure 1A). Meanwhile, the RTCA CIM plate is prepared, firstly by coating the porous membrane interface with matrix and then a confluent monolayer of human mesothelial cells (Figure 1B). Once formed, spheroids are then transferred to the prepared CIM plate. Harvested cancer spheroids are plated on top of the LP9 monolayer and assessed as described below. Images under standard phase microscopy (or under fluorescent microscopy, if the optional cell labeling step was followed) of parallel cultures are taken periodically to aid in the interpretation of the RTCA data (Figure 1C).

It is informative to assess both the basal level of invasion of a cancer cell line, as well as chemoattractant-induced invasion. Typically, basal invasiveness is measured by the addition of SFM to both the upper and lower chambers. Complete media (10\% FBS), which contains many potential chemoattractants, is used in the current example to examine 'chemoattractant-induced' invasion (Figure 2). Parallel studies of LP9 mesothelial cells alone confirmed that these cells are minimally invasive over 2 days under either basal or 'chemoattractant' induced conditions and thus were a good cell type to use in co-culture assays with ovarian cancer cells (Figures $2 \mathrm{~A}$ and $2 \mathrm{~B}$ ). In contrast, ovarian cancer spheroids generated using any of the three cell lines exhibited the capacity to invade toward a chemoattractant (FBS) (Figures 2A-2C). The major advantage of using the RTCA real time instruments over traditional endpoint assays is that changes in cell/spheroid behavior can be quantified 
over time. Over the 2 day assay, the KGN, OVCA429, and OVCA433 cell lines all exhibited the capacity to invade toward a chemoattractant (indicated by increasing cell indices) but low basal levels of invasion (Figure 2C). The cell lines exhibited similar rates of invasion, as indicated by the parallel slopes of the curves (Figure 2C); however, the OVCA429 curve exhibited a higher upper asymptote, which indicated a higher maximal level of invasion compared to the other cell lines. Furthermore, the cell lines exhibited differences in their times to onset of invasion, with the KGN cells quickly invading the cell and matrix barriers and the OVCA433 and OVCA429 cells taking 3-5x longer to invade, respectively (Figure 2D). Importantly, their behaviors at the onset of invasion were not predictive of their overall capacity for invasion (Figures 2C and 2D). This suggests different inherent capabilities of the cancer cell lines but also may indicate that different factors may regulate early and late invasive behaviors of spheroids.

\section{A}

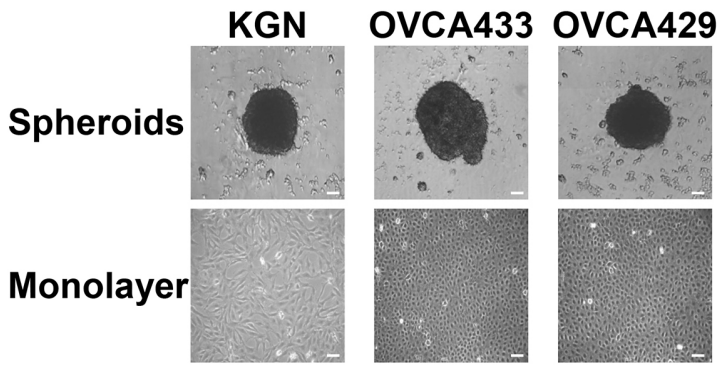

B

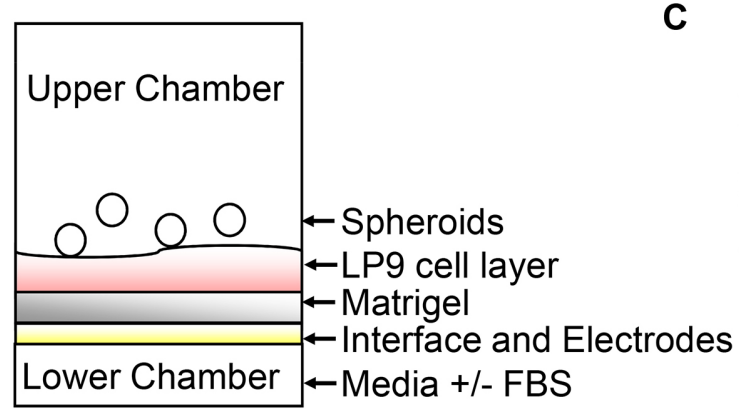

\section{Spheroids on LP9 cells}

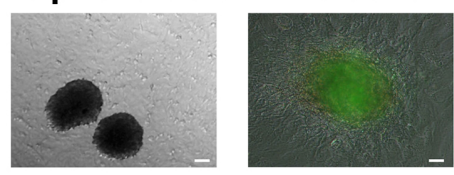

Figure 1. Spheroid generation and model of experimental set up. A: Images under phase contrast microscopy of spheroids forming in methylcellulose/media (top) and of the matching cell line grown as a monolayer (bottom). B: Schematic showing the RTCA 2 chambered CIM plate well set up, in which pre-formed ovarian cancer spheroids are plated on top of a monolayer of an LP9 mesothelial layer/matrix barrier in the upper chamber and media \pm FBS as a chemoattractant is added to the lower chamber. Electrodes underneath the interface of the 2 chambers measure increasing electrical impedance as more cells invade through the barriers to the lower chamber. C: Images of KGN spheroids on top of the LP9 monolayer under phase contrast microscopy $($ left $)$ or fluorescence microscopy (right). Scale bars $=100 \mu \mathrm{m}$. Please click here to view a larger version of this figure. 
A

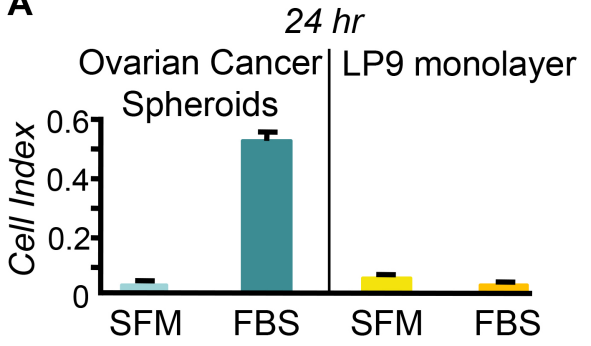

C

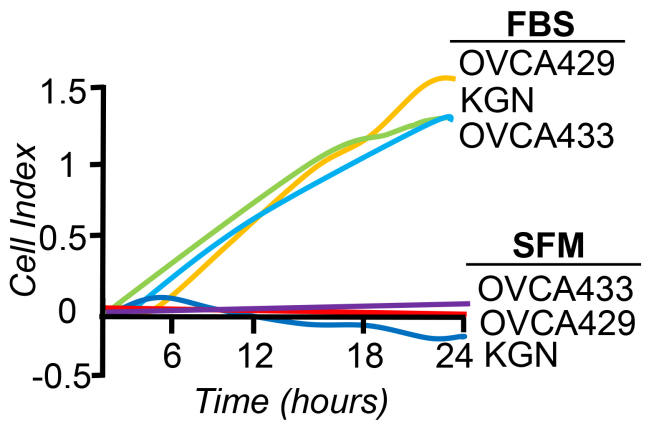

B

D
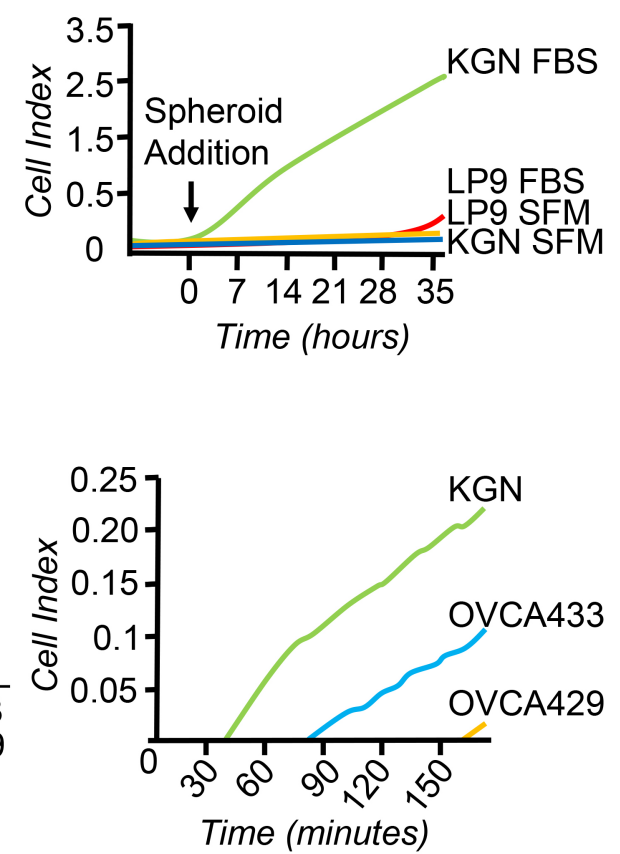

Figure 2. Real time ovarian cancer spheroid invasion data. A - B: Representative results from an RTCA invasion assay conducted with and without FBS in the bottom chamber of a CIM plate well. KGN cell invasion is compared to LP9 mesothelial cells. Results are shown as mean \pm SD Cell Index from triplicate wells at the $24 \mathrm{hr}$ timepoint (A) and over an entire two day assay period (B). C - D: A comparison of the invasive capacity of KGN, OVCA429, and OVCA433 cell lines depicted over a $24 \mathrm{hr}$ period (C) and in more detailed fashion over a $2.5 \mathrm{hr}$ time period (D).

\section{Discussion}

Invading ovarian cancer cells interact with a variety of cell types at the surface of the peritoneum, including fibroblasts, adipocytes, and mesothelial cells, and bidirectional communication between the cancer cells and peritoneal cells is thought to play key roles in driving the metastatic process ${ }^{2}$. Once mastered, the protocol described herein is readily adaptable to the study of these interactions within the peritoneal microenvironment, e.g., through the addition of exogenous cytokines, growth factors, or specific inhibitors to the upper or lower chambers of the CIM plate well or genetic manipulation of the cancer or peritoneal cell lines. Furthermore, this method can be used with primary ovarian tumor cells harvested freshly from malignant ascites and/or primary peritoneal cells to gain direct insights into the regulatory signals and cellular interactions which govern the establishment of a metastatic lesion within the peritoneal cavity ${ }^{2}$.

Use of the RTCA instrument to measure the invasive capacity of single cells or spheroids offers several advantages over conventional single endpoint assays and time-lapse image analyses. The RTCA instrument measures cellular invasion at defined intervals continuously over the course of an assay, which can be hours or days. This enables the determination of changes in the rate of invasion over time, which overcomes the limitations of single endpoint assays. For example, examining the invasion data from the different cancer cell lines at a single endpoint (Figure 2), e.g., $3 \mathrm{hr}$, might have led to the erroneous conclusion that KGN and OVCA433 cells were far more invasive than OVCA429 cells. Another advantage of this technology is that the RTCA instrument measures changes in electrical impedance. This means that assays can be run without labeling cells with an exogenous agent, which could have unintended effects on cell phenotype or function. This becomes particularly important when studying primary ovarian cancer cells derived from malignant ascites, with which it is imperative to keep manipulations to a minimum so as to avoid introducing molecular and phenotypic changes which are not reflective of their in vivo nature ${ }^{2}$. Furthermore, use of the RTCA instrument enables the collection of quantitative data in real time, which not only avoids the time consuming analyses associated with time-lapse microscopy but also enables the rapid determination of key experimental windows for assay optimization. This is particularly useful when comparing the effects of a range of factors on spheroid invasion, as different factors may exert their maximal effects at different timepoints or with greatly different profiles over time.

Although this protocol is amenable to alterations (e.g., use of different ECM matrix, different cancer cell lines, or different target cells), if doing so, it is important to note that various experimental conditions must first be optimized. For example, prior to commencing studies of spheroid invasion, the optimal number of cancer cells/CIM plate well should firstly be determined by assay of cell number titrations in monolayer culture. The optimal number of spheroids to use per well is then based on this analysis. For example, in the current method, spheroids comprise approximately 3,000 cells each when first generated. If initial cell number titrations indicate that 30,000 cells in monolayer are optimal for detection in invasion analyses, then 10 spheroids are added per CIM plate well. Furthermore, when conducting co-culture experiments, it is important to study the behaviors of the 'target' cell monolayer separately in order to determine whether these cells are inherently invasive, as this could confound the results. In the example demonstrated, cancer spheroids are plated on top of an LP9 cell monolayer, with and without FBS added to the bottom well as a chemoattractant. In parallel, LP9 cells are cultured alone, under each treatment condition.

Similarly, when studying the invasive capacity of cells and spheroids, it is also important to examine their migratory capacities as well in order to distinguish the two behaviors (i.e. invasion requires the proteolytic digestion of an ECM barrier while migration does not). To do this, omit the 
ECM barrier (Steps 3.1.1 - 3.1.3) and conduct the assay in parallel with the ECM barrier in place. The latter 'invasion' measure can be corrected to the former 'migration' measure, if desired. Finally, it is important to note that the information gained from measures of electrical impedance is limited in scope: once cells have invaded into the bottom chamber, changes in their attachment, spreading, and proliferation on the underside of the membrane may contribute to changes in impedance readings. Therefore, this methodology is most informative when used in conjunction with other assays of spheroid cell viability, adhesion, migration, and morphology in order to comprehensively assess spheroid cell behaviors. For example, ideally, in order to fully understand spheroid-mesothelial interactions, separate co-cultures should also be imaged periodically under standard phase microscopy so that qualitative assessments of spheroid morphology and health can be made in parallel with quantitative RTCA assays of invasion. If the optional labeling step is performed, then the behaviors of single cancer cells can be determined under fluorescent microscopy as they disaggregate from the spheroid and interact with the unlabeled mesothelial cells. An added benefit of fluorescently labeling the cancer cells when co-culturing with a second cell type is that it is then possible to confirm that only the cancer cells have invaded through the cell and ECM barriers.

In summary, this method represents a high throughput quantitative analysis of ovarian cancer spheroid invasion of mesothelial and ECM barriers Through the addition of exogenous cytokines, growth factors, or specific inhibitors to the upper or lower chambers of the CIM plate well, this method enables a rapid, determination of factors which regulate the interactions between ovarian cancer spheroid cells invading through mesothelial and matrix barriers over time.

\section{Disclosures}

Open access fee paid for by ACEA Biosciences, Inc. and MIMR-PHI Institute of Medical Research.

\section{Acknowledgements}

This work was supported by a CASS Foundation Science and Medicine Grant; (MB); a National Health \& Medical Research Council of Australia Project Grant (KLS, 338516); and by the Victorian Government's Operational Infrastructure Support Program (Australia).

\section{References}

1. Ferlay, J., et al. Estimates of worldwide burden of cancer in 2008: GLOBOCAN 2008. Int J Cancer. 127, 2893-2917, doi:10.1002/ijc.25516 (2010).

2. Ahmed, N., \& Stenvers, K. Getting to know ovarian cancer ascites: opportunities for targeted therapy- based translational research. Frontiers in Oncology. 3, doi:10.3389/fonc.2013.00256 (2013).

3. Karst, A. M., \& Drapkin, R. Ovarian cancer pathogenesis: a model in evolution. J Oncol. 2010, 932371, doi:10.1155/2010/932371 (2010)

4. Lengyel, E. Ovarian cancer development and metastasis. Am J Pathol. 177, 1053-1064, doi:S0002-9440(10)60160-5 [pii]10.2353/ ajpath.2010.100105 (2010).

5. Shield, K., Ackland, M. L., Ahmed, N., \& Rice, G. E. Multicellular spheroids in ovarian cancer metastases: Biology and pathology. Gynecol Oncol. 113, 143-148, doi:S0090-8258(08)00974-8 [pii]10.1016/j.ygyno.2008.11.032 (2009).

6. Hudson, L. G., Zeineldin, R., \& Stack, M. S. Phenotypic plasticity of neoplastic ovarian epithelium: unique cadherin profiles in tumor progression. Clin Exp Metastasis. 25, 643-655, doi:10.1007/s10585-008-9171-5 (2008).

7. Burleson, K. M., Boente, M. P., Pambuccian, S. E., \& Skubitz, A. P. Disaggregation and invasion of ovarian carcinoma ascites spheroids. $J$ Transl Med. 4, 6, doi:1479-5876-4-6 [pii]10.1186/1479-5876-4-6 (2006).

8. Burleson, K. M., et al. Ovarian carcinoma ascites spheroids adhere to extracellular matrix components and mesothelial cell monolayers. Gynecol Oncol. 93, 170-181, doi:10.1016/j.ygyno.2003.12.034 S0090825803009508 [pii] (2004).

9. Burleson, K. M., Hansen, L. K., \& Skubitz, A. P. Ovarian carcinoma spheroids disaggregate on type I collagen and invade live human mesothelial cell monolayers. Clin Exp Metastasis. 21, 685-697 (2004).

10. Kenny, H. A., Kaur, S., Coussens, L. M., \& Lengyel, E. The initial steps of ovarian cancer cell metastasis are mediated by MMP-2 cleavage of vitronectin and fibronectin. J Clin Invest. 118, 1367-1379, doi:10.1172/JCI33775 (2008).

11. Kenny, H. A., Nieman, K. M., Mitra, A. K., \& Lengyel, E. The First Line of Abdominal Metastatic Attack: Breaching the Mesothelial Cell Layer. Cancer Discov. 1, 100-102, doi:10.1158/2159-8290.CD-11-0117 (2011).

12. Hattermann, K., Held-Feindt, J., \& Mentlein, R. Spheroid confrontation assay: a simple method to monitor the three-dimensional migration of different cell types in vitro. Ann Anat. 193, 181-184, doi:S0940-9602(11)00035-5 [pii] 10.1016/j.aanat.2010.12.005 (2011).

13. Laib, A. M., et al. Spheroid-based human endothelial cell microvessel formation in vivo. Nat Protoc. 4, 1202-1215, doi:nprot.2009.96 [pii] 10.1038/nprot.2009.96 (2009).

14. Latifi, A., et al. Isolation and characterization of tumor cells from the ascites of ovarian cancer patients: molecular phenotype of chemoresistant ovarian tumors. PLoS One. 7, e46858, doi:10.1371/journal.pone.0046858 PONE-D-12-20118 [pii] (2012).

15. Davidowitz, R. A., Iwanicki, M. P., \& Brugge, J. S. In vitro mesothelial clearance assay that models the early steps of ovarian cancer metastasis. J Vis Exp. (60), doi:10.3791/3888 (2012).

16. Iwanicki, M. P., et al. Ovarian cancer spheroids use myosin-generated force to clear the mesothelium. Cancer Discov. 1, 144-157, doi:10.1158/2159-8274.CD-11-0010 (2011).

17. Nishi, Y., et al. Establishment and characterization of a steroidogenic human granulosa-like tumor cell line, KGN, that expresses functional follicle-stimulating hormone receptor. Endocrinology. 142, 437-445 (2001).

18. Xu, F., et al. The outcome of heregulin-induced activation of ovarian cancer cells depends on the relative levels of HER-2 and HER-3 expression. Clin Cancer Res. 5, 3653-3660 (1999). 